\title{
Editorial
}

\section{Influenza and Pneumococcal Vaccination: How Far We Have Come and How to Go Farther}

\author{
Henry M. Wu, MD; Elias Abrutyn, MD
}

The recent outbreak of avian influenza in Asia is a timely reminder of the ever present possibility of a human influenza pandemic in the near future. ${ }^{1}$ Work continues to develop vaccines effective against emergent influenza strains, but another component of effective prevention and infection control programs is the ability to administer vaccine in a timely and efficient manner. Even in the absence of a pandemic, influenza-associated respiratory and circulatory illness results in more than 200,000 hospitalizations and 36,000 deaths each year in the United States, according to recent estimates. ${ }^{2,3}$ These numbers suggest that the routine administration of influenza vaccine to targeted populations remains a major challenge.

The efficacy of influenza and pneumococcal vaccines in preventing illness is well established, ${ }^{4,5}$ and during the past decade substantial progress has been made in increasing influenza and pneumococcal vaccination coverage in targeted populations. Estimates of influenza vaccination levels among individuals 65 years and older based on the National Health Interview Survey (NHIS) showed an increase from 33\% in 1989 to $66 \%$ in the 2001-2002 season ${ }^{4,6}$ NHIS estimates for pneumococcal vaccine coverage in this population also increased (from $15 \%$ in 1989 to $54 \%$ in 2001) ${ }^{6.7}$ However, influenza vaccination rates in all other targeted adult populations remained well below $50 \%$ in the 2001-2002 season, including healthcare workers (38\%) and adults 18 to 49 years old with high-risk conditions (23\%). ${ }_{4}^{4}$ Influenza vaccination rates among children at increased risk for influenza complications have also been low. ${ }^{4}$ This year, the Advisory Committee on Immunization Practices (ACIP) extended use of influenza vaccine by recommending vaccination for healthy children 6 to 23 months old and close contacts of children 0 to 23 months old. ${ }^{4}$ These fig- ures suggest that despite some progress, targeted populations, including many workers and patients of healthcare institutions, still remain vulnerable.

In this issue of Infection Control and Hospital Epidemiology, several articles address the challenges we face in further increasing vaccination levels among targeted populations. ${ }^{8-12}$ The study by Coyle and Currie is a welcome addition to the literature about interventions to increase pneumococcal vaccination rates among hospitalized patients. ${ }^{8}$ This study compared a standing orders protocol with computerized physician reminders and with routine practice in a prospective trial. The study found a clear superiority of standing orders over physician reminders, a finding consistent with previous studies ${ }^{13}$ and supportive of the ACIP recommendation for healthcare institutions to implement standing orders programs to increase pneumococcal and influenza vaccine coverage of high-risk individuals. ${ }^{4,5}$

Two studies examine employee influenza vaccination policies at healthcare institutions. ${ }^{9,10}$ In a large survey of healthcare institutions in North Carolina, Goldstein et al. found that only $38 \%$ reported having formal written policies regarding employee influenza vaccination. ${ }^{9}$ The lowest rates were found in assisted living facilities and dialysis centers. The authors also noted that barriers to increasing levels of influenza vaccination may differ depending on the type of institution, further emphasizing the need to adapt vaccination interventions to the specific needs of an individual institution. The article by Bryant et al. describes a survey of influenza vaccination policies among several pediatric hospitals. ${ }^{10}$ The median employee vaccination rate reported by infection control practitioners was $43 \%$ in this voluntary survey. A point-prevalence survey of employ- 
ees in high-risk units (intensive care units and oncology wards) revealed a vaccination rate of $53 \%$. The reason most commonly cited for accepting vaccination was "a desire to protect one's patients." This indicates that when offering influenza vaccination, we should always emphasize the altruistic nature of accepting it. Reasons cited for refusing vaccination were similar to those reported in the past, including beliefs that the vaccine causes influenza, perceived side effects, inconvenience, and a fear of vaccination. ${ }^{14}$ With the exception of a fear of vaccination, all of these barriers potentially can be overcome with interventions that include education along with facilitating employee access to vaccines. With the approval of an intranasal, live attenuated influenza vaccine (LAIV) in 2003, there is hope that, even among those who fear shots, vaccination rates can be improved. However, use in the healthcare setting must proceed cautiously because of concern about transmission of vaccine virus to contacts of vaccinees. Those in close contact with severely immunosuppressed individuals should not receive the vaccine. ${ }^{4}$

Two other articles in this issue of Infection Control and Hospital Epidemiology evaluate specific hospital employee influenza vaccination interventions. ${ }^{11,12}$ Both studies integrated a mobile cart program with educational campaigns. In the study reported by Sartor et al., the overall employee vaccination rate increased from 7\% in 1999 to $32 \%$ in $2000 .{ }^{11}$ Although the effect was sustained for the next 2 years, the rate did not increase fur ther, emphasizing the importance of ongoing assessments of vaccination programs to target areas for further improvement. An interesting finding was that vaccination of the chief within a specific unit was associated with higher vaccination rates among medical staff. Although the causal pathway of this association is unclear, the importance of leadership establishing clearly the desired behavior should not be underestimated.

The vaccination intervention described by Salgado et al. resulted in a gradual increase of hospital employee vaccination from a baseline of $4 \%$ during the $1987-1988$ season to $67 \%$ during $1999-2000 .^{12}$ Not surprisingly, the increase in vaccination coverage corresponded with a decrease in the relative frequency of laboratory-confirmed influenza cases among healthcare workers. Moreover, the study showed a significant decrease in the relative frequency of hospitalacquired influenza cases among patients, which appeared associated with the increased employee vaccine coverage. Although the first year of the vaccination intervention coincided with the implementation of intensified influenza infection control policies, the continued decline of nosocomial influenza cases during several years supports the assertion that increased employee vaccine coverage was a major factor. This finding adds further evidence that healthcare employee vaccination can benefit patients. Previous studies have associated increased healthcare worker vaccination coverage with decreased mortality among nursing home residents. ${ }^{15.16}$ The comprehensive approach to influenza control as well as sustained efforts over the long-term were likely factors in the success of this intervention.
Infection control practitioners and hospital epidemiologists can successfully prevent pneumococcal and influenza infections in their institutions with a multifaceted approach, including vaccination, education, and traditional infection control methods. Effective surveillance is critical, not only to detect new cases, but also to assist public health authorities in detecting new disease patterns or emerging pathogens. Unfortunately, the vaccination of workers and high-risk individuals in healthcare institutions remains a significant obstacle. Furthermore, there have been warnings of a likely shortage of influenza vaccine this season secondary to manufacturing problems at Chiron, one of two suppliers of the inactivated vaccine to the United States. ${ }^{17}$ With any vaccine shortage, strict following of Centers for Disease Control and Prevention recommendations will be absolutely imperative. These articles in this issue of Infection Control and Hospital Epidemiology add to the body of experience that will assist infection control practitioners and hospital administrators in improving results of their vaccination programs. Educational campaigns, mobile carts, standing orders, and other evidencebased interventions are among the potential tools that can be adapted to individual needs. The price of LAIV recently has been reduced significantly to encourage its use, ${ }^{18}$ and LAIV may play an important role in vaccination programs as we gain experience in its use. Local legislation may also assist the implementation and acceptance of vaccination programs. In our own state, for example, a law was recently signed requiring healthcare facilities to offer pneumococcal and influenza vaccinations to patients 65 years and older.

Influenza-associated hospitalizations and deaths in the United States have increased substantially during the past decade, and this appears to be due to several factors, including our progressively aging population.,3 Further increases are therefore likely unless we improve our vaccination coverage. We have the tools to accomplish this, and with an integrated approach we will not only fulfill our mission to prevent infections in our institutions, but also contribute significantly to the prevention of pneumococcal and influenza disease in our communities at large.

\section{REFERENCES}

1. Katz JM. Preparing for the next influenza pandemic. ASM News 2004;70:412-419.

2. Thompson WW, Shay DK, Weintraub E, et al. Mortality associated with influenza and respiratory syncytial virus in the United States. JAMA 2003;289:179-186.

3. Thompson WW, Shay DK, Weintraub E, et al. Influenza-associated hospitalizations in the United States. JAMA 2004;292:1333-1340.

4. Harper SA, Fukuda K, Uyeki TM, Cox NJ, Bridges CB. Prevention and control of influenza: recommendations of the Advisory Committee on Immunization Practices (ACIP). MMWR 2004;53(RR-6):1-40.

5. Prevention of pneumococcal disease: recommendations of the Advisory Committee on Immunization Practices (ACIP). MMWR 1997;46(RR-8):124.

6. Influenza and pneumococcal vaccination coverage levels among persons aged $>$ or $=65$ years: United States, 1973-1993. MMWR 1995;44:506-507, 513-505.

7. Influenza and pneumococcal vaccination levels among persons aged $>$ or = 65 years: United States, 2001. MMWR 2002;51:1019-1024.

8. Coyle CM, Currie BP. Improving the rates of inpatient pneumococcal vaccination: impact of standing orders versus computerized reminders to physicians. Infect Control Hosp Epidemiol 2004;25:904-907. 
9. Goldstein AO, Kincade JE, Gamble G, Bearman RS. Policies and practices for improving influenza immunization rates among healthcare workers. Infect Control Hosp Epidemiol 2004;25:908-911.

10. Bryant KA, Stover B, Cain L, Levine GL, Siegel J, Jarvis WR. Improving influenza immunization rates among healthcare workers caring for highrisk pediatric patients. Infect Control Hosp Epidemiol 2004;25:912-917.

11. Sartor C, Tissot-Dupont H, Zandotti C, Martin F, Roques P, Drancourt M. Use of a mobile cart influenza program for vaccination of hospital employees. Infect Control Hosp Epidemiol 2004;25:918-922.

12. Salgado CD, Giannetta ET, Hayden FG. Farr BM. Preventing nosocomial influenza by improving the vaccine acceptance rate of clinicians. Infect Control Hosp Epidemiol 2004;25:923-928.

13. Fedson DS, Houck P, Bratzler D. Hospital-based influenza and pneumococcal vaccination: Sutton's Law applied to prevention. Infect Control
Hosp Epidemiol 2000;21:692-699.

14. Salgado CD, Farr BM, Hall KK, Hayden FG. Influenza in the acute hospital setting. Lancet Infect Dis 2002;2:145-155.

15. Potter J, Stott DJ, Roberts MA, et al. Influenza vaccination of health care workers in long-term-care hospitals reduces the mortality of elderly patients. J Infect Dis 1997;175:1-6.

16. Carman WF, Elder AG, Wallace LA, et al. Effects of influenza vaccination of health-care workers on mortality of elderly people in long-term care: a randomised controlled trial. Lancet 2000;355:93-97.

17. Centers for Disease Control and Prevention. Interim influenza vaccination recommendations, 2004-05 influenza season. MMWR 2004;53:923924.

18. Grassia T. Price of FluMist slashed, partnership announced. Infectious Disease News. August 2004:14-15. wary shectonling ont www shea-online.or www shea-online org www shea-online or www shea-onine.org www shea-ordine on www shea-orline org www shea-online or www shea-online org wyrw shea-online org why shea oving or

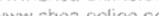
ww shed ontine or wrw.shea-ontine or wWW shea ontine. of ww shearonline or ww shea online or www shea-online.org Ww silea-online. or www.sheâ-brline. org ww. shea.orline.org Wwishea online. or www shea online.ong www sheer ontine. of www shearonline, or sww shea-online. op Fww shearonline of ww shea-olline.o: ww shea-online on ww. Shea ondine. of www shea-online or www sheat-online. Or ww shed orthe.or www shea-online of vww shea online org wwo shearonline ors

\section{Same Website Address, New Design}

The Site Map allows you to locate your favorite links on the site at a moment's glance.

The Society for Healthcare Epidemiology (SHEA) is pleased to announce the launch of its redesigned website, featuring a more professional, informative, and accessible design Enjoy navigating through our links, articles, updates, and archives.

\section{Please note several new features:}

The Search feature allows you to query the site.

The Home page provides two news clips and links to the most recent SHEA News articles and Epidemiology News articles.

The Membership Information section allows you to update your own information, renew your membership, and search SHEA's membership.
By logging in as a SHEA Member, you will have access to the Infection Control and Hospital Epidemiology article archives, the abstracts from the 2001 and 2002 Annual Meeting, and Member Only News.

\section{Login Procedure:}

To log in, please click on the Member Login link at the top, right corner of each webpage. Enter the most recent e-mail address you sent to SHEA in the User Email field, and your member number in the Password field. You can click on "Forgot Your Password?" to have your member number sent to your e-mail address. If you have any questions

(703) 684-1006 or sheahq@shea-online.org. about the site, please contact us at: 